\title{
PROGRESSIVE OUTER RETINAL NECROSIS (PORN) IN AIDS PATIENTS: A DIFFERENT APPEARANCE OF VARICELLA-ZOSTER RETINITIS
}

\author{
C. E. PAVESIO ${ }^{1}$, S. M. MITCHELL ${ }^{1,2}$ K. BARTON ${ }^{1}$, S. D. SCHWARTZ ${ }^{1,3}$, H. M. A. TOWLER ${ }^{1}$ \\ and S. LIGHTMAN ${ }^{1}$ \\ London and Los Angeles
}

\begin{abstract}
SUMMARY
Retinal infections caused by the varicella-zoster virus (VZV) have been reported in immunocompetent and immunocompromised individuals. Two cases of a VZV. related retinitis are described with the characteristic features of the recently described progressive outer retinal necrosis (PORN) syndrome. Both patients suffered from the acquired immunodeficiency syndrome (AIDS) with greatly reduced peripheral blood $\mathrm{CD4}^{+} \mathrm{T}$ lymphocyte counts, and presented with macular retinitis without vitritis. The disease was bilateral in one case and unilateral in the other. The clinical course was rapidly progressive with widespread retinal involvement and the development of rhegmatogenous retinal detachment with complete loss of vision in the affected eyes despite intensive intravenous antiviral therapy. VZV DNA was identified in vitreous biopsies, by molecular techniques based on the polymerase chain reaction (PCR), in both patients. At present, the use of very high-dose intravenous acyclovir may be the best therapeutic option in these patients for whom the visual prognosis is poor. Intravitreal antiviral drugs could also contribute to the management of these cases.
\end{abstract}

Opportunistic infections of the retina are common in patients with acquired immunodeficiency syndrome (AIDS), and may be caused by viruses, bacteria, fungi and other parasites. ${ }^{1}$ Pneumocystis carinii choroiditis is an example of a new infection not encountered prior to the beginning of the AIDS

From: ${ }^{1}$ Uveitis and Acquired Immunodeficiency Syndrome Clinics, Moorfields Eye Hospital, and Department of Clinical Ophthalmology, Institute of Ophthalmology, London; ${ }^{2}$ Division of Virology, Department of Medical Microbiology, University College, London Medical School, London; ${ }^{3} J u l e s$ Stein Eye Institute, UCLA School of Medicine, Los Angeles, California, USA.

Correspondence to: Professor S. Lightman, Department of Clinical Ophthalmology, Moorfields Eye Hospital, City Road, London EC1V 2PD, UK. epidemic, but the majority of retinal infections which develop in AIDS patients are also known to occur in immunocompetent patients. ${ }^{2}$ Viral infections are the most frequent, especially cytomegalovirus (CMV) retinitis, which occurs in $20-25 \%$ of these patients. ${ }^{2}$ Other viruses of the herpes family also cause retinal infections, such as acute retinal necrosis, associated with both varicella-zoster virus (VZV) and herpes simplex virus (HSV). ${ }^{3}$ A syndrome has recently been described $^{4,5}$ of progressive outer retinal necrosis (PORN) in patients with AIDS. In the first report herpes virus particles were identified on retinal biopsy in one of two cases and by the polymerase chain reaction (PCR) in the other. In the second report, VZV was cultured from chorioretinal specimens in two cases, from vitreous aspirate in one case and from the outer retinas of enucleated eyes in two further cases.

This paper presents two cases of PORN diagnosed in the United Kingdom.

\section{CASE REPORTS}

Case 1

A 33-year-old white homosexual male presented, in November 1992, with a 14 day history of acute loss of vision in his left eye. He had not experienced pain, redness or photophobia and he had no symptoms from his right eye. His past medical history included a positive human immunodeficiency virus (HIV) antibody test in July 1991, thoracic shingles in 1988 with a recurrence in 1992, and cryptococcal meningitis in February 1992 with two recurrences, initially in July 1992 and subsequently 2 weeks prior to his ophthalmic presentation.

There had been no previous abnormalities in his ophthalmic history and his $\mathrm{CD} 4^{+} \mathrm{T}$ lymphocyte count at presentation was 1 cells $/ \mathrm{mm}^{3}$ (normal range: $750-1000$ cells $/ \mathrm{mm}^{3}$ ). The corrected visual acuity in

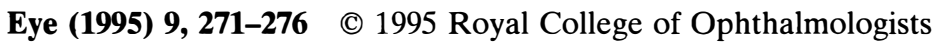




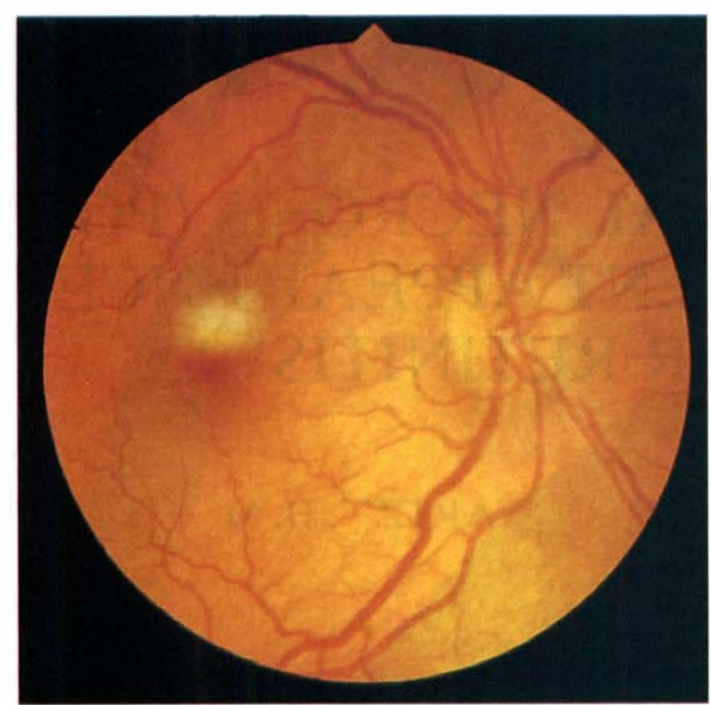

(a)

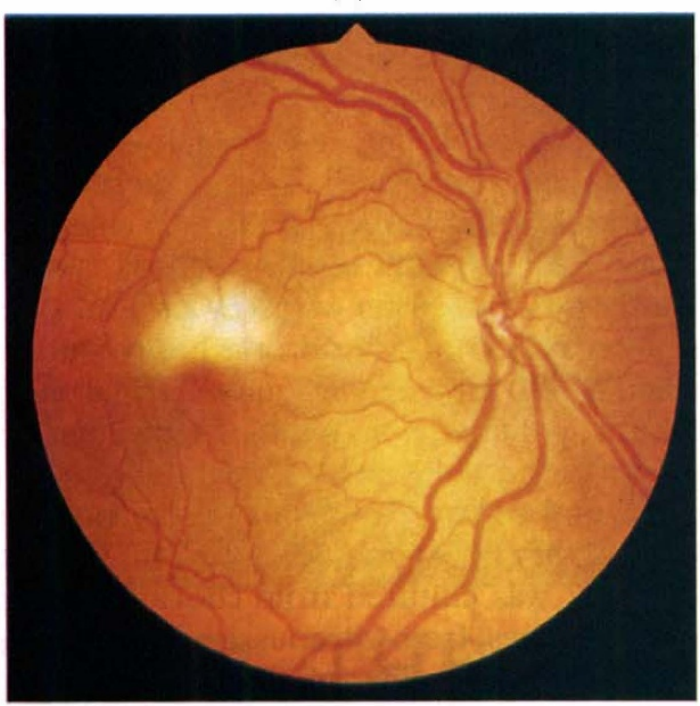

(c)

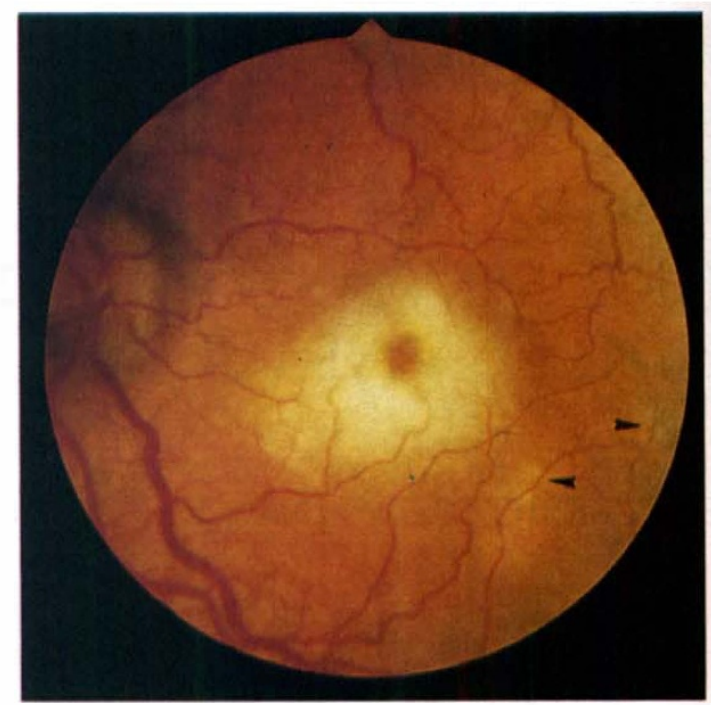

(b)

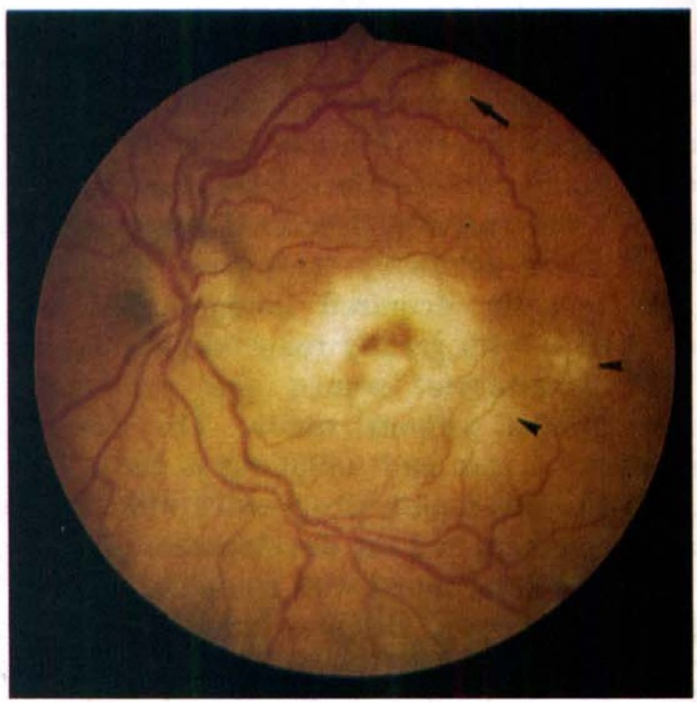

(d)

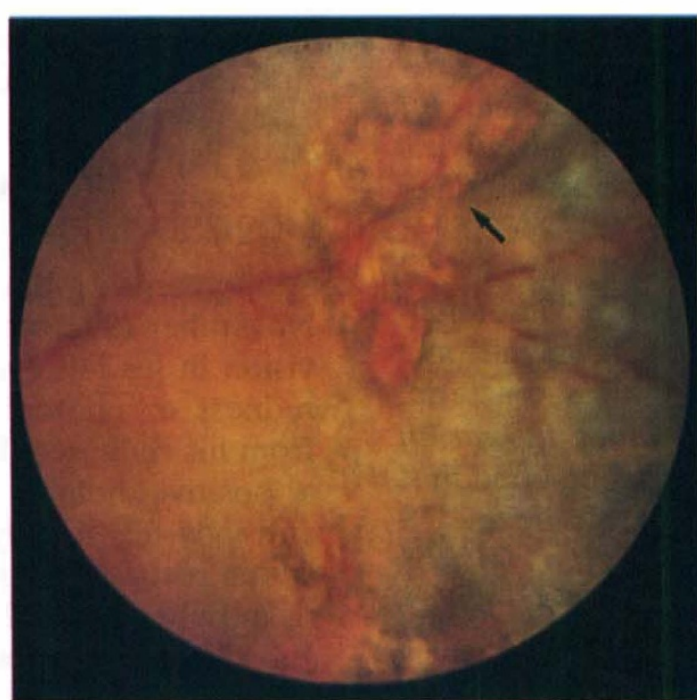

(e)

Fig. 1. Patient 1. (a) Superior parafoveal lesion (one-third to one-half disc diameter in the right eye. The margins of the optic disc are elevated. There is no evidence of vasculitis or vitritis. (b) Large central area of retinitis with a cherry-red spot appearance in the left eye. Note the presence of a few early satellite lesions (arrowheads). (c) Parafoveal lesion in the right eye has enlarged (one disc diameter) in spite of therapy. (d) Central area of retinitis in the left eye has enlarged. Satellite lesions have increased in size and started to merge with the main lesion (arrowheads). New satellite lesions can also be seen (arrow). (e) Peripheral retina of the left eye. Some areas of apparently uninvolved retina can be seen along some retinal vessels (arrow). 
his right eye was $6 / 6$, and $1 / 60$ in his left eye. The anterior segments were both quiet and the intraocular pressures were $10 \mathrm{mmHg}$ in each eye. Examination of the retina revealed a parafoveal, white lesion of the outer retina in the right eye (Fig. 1a). There was a white lesion at the left macula, one disc diameter in size, with a central cherry-red spot (Fig. 1b). Both optic discs were swollen but the periphery of each retina was completely normal.

The differential diagnosis of bilateral outer retinitis with swollen optic discs includes atypical presentation of CMV retinitis, cryptococcal retinitis (with possible reactivation of meningitis), toxoplasma retinitis, zoster retinitis and primary central nervous system lymphoma. A lumbar puncture and computed tomography (CT) scan of the brain were therefore performed, but did not reveal active intracranial infection or evidence of raised intracranial pressure. A vitreous biopsy taken at presentation revealed the presence of VZV DNA only, with no evidence of either CMV or HSV-1 DNA (see Methods).

The retinitis was initially managed with intravenous (IV) acyclovir ( $10 \mathrm{mg} / \mathrm{kg}$ three times per day) in combination with IV foscarnet $(60 \mathrm{mg} / \mathrm{kg}$ three times per day). The lesions in both eyes progressed despite therapy, with the appearance of new satellite lesions which became confluent around the central lesions in both eyes (Figs. 1c, d). After 1 week visual acuity of the right eye was reduced to 6/12 due to enlargement of the central lesion. On the tenth day, multiple areas of retinitis developed in the mid-periphery and periphery of the left eye, which were shortly followed by the development of a supero-temporal retinal detachment. The vitreous was attached and no cells could be seen in either eye.

Argon laser photocoagulation was undertaken in an attempt to limit the progression of the left retinal detachment. An intravitreal injection of $0.2 \mathrm{mg} /$ $0.1 \mathrm{ml}$ of acyclovir was given in the right eye. After 48 hours the dose of acyclovir was increased to 20 $\mathrm{mg} / \mathrm{kg}$ three times per day IV, and daily injections of intra vitreal acyclovir for 1 week were commenced.

One week later, the lesions in both eyes were less active and the optic discs were no longer swollen; the retina at the posterior pole remained attached in the left eye and clear, non-opacified areas of retina could be seen along the retinal vessels in the periphery of this eye (Fig. 1e). After 3 weeks, the dose of IV acyclovir was reduced to $15 \mathrm{mg} / \mathrm{kg}$ three times per day, and then to $10 \mathrm{mg} / \mathrm{kg}$ three times per day after a further week.

Problems with the patient's central venous indwelling catheter led to replacement of the IV treatment with oral acyclovir $800 \mathrm{mg}$ five times per day. After another 2 weeks, the retinitis reactivated in both eyes with the development of peripheral lesions in the right eye, and progression of the retinal detachment in the left eye. Intravenous therapy was therefore reintroduced. However, a retinal detachment developed in the right eye and the left eye lost light perception due to continued disease resulting in complete retinal detachment.

The right retina was re-attached following vitrectomy, retinotomy, endolaser and silicone oil, resulting in a visual acuity of $6 / 60$ and a very restricted visual field. At this stage the patient elected to discontinue with IV treatment and when next seen this eye had also lost light perception. He subsequently decided to stop all medical treatment and died within a few weeks. Authorisation to obtain his eyes at post-mortem was not obtained.

\section{Case 2}

A 37-year-old white homosexual male presented with a 10 day history of acute, profound loss of vision in his right eye, without any other symptoms. He was HIV antibody positive and had been diagnosed as having AIDS 1 year before developing ocular symptoms. Left CMV retinitis had been diagnosed 5 days previously and he had commenced an induction course of IV ganciclovir $(5 \mathrm{mg} / \mathrm{kg}$ twice a day). There were no previous ophthalmic problems. His ophthalmic presentation coincided with a sudden drop in his peripheral blood $\mathrm{CD}^{+} \mathrm{T}$ lymphocytes to 14 cells $/ \mathrm{mm}^{3}$.

On examination of the eyes his visual acuity was light perception with accurate projection in the right eye and $6 / 6$ in the left eye. There was a low-grade right anterior uveitis and a mild bilateral vitritis. His right intraocular pressure was $5 \mathrm{mmHg}$ and the left was $8 \mathrm{mmHg}$. The right fundus showed a large white lesion at the right macula (Fig. 2a), with multiple, white, outer retinal lesions in the periphery and midperiphery.

In the left eye there were two areas of typical active CMV retinitis in the supero-nasal and inferotemporal quadrants but no other retinal lesions. Both optic discs appeared normal. Cerebrospinal fluid was normal on lumbar puncture, and VZV DNA was identified by PCR from a vitreous biopsy of the right eye, with no evidence of CMV or HSV-1 DNA. Vitreous biopsy from the left eye revealed only CMV DNA. A diagnosis of PORN in the right eye was considered "and high-dose IV acyclovir $(20 \mathrm{mg} / \mathrm{kg}$ three times per day) was commenced. A course of four intravitreal injections of acyclovir $(0.2 \mathrm{mg} /$ $0.1 \mathrm{ml}$ ) was given during the first 2 weeks of treatment.

The peripheral lesions became more prominent in the right eye, and laser demarcation was not attempted due to the widespread distribution of retinal lesions. An area of retinal traction developed in the supero-temporal quadrant of the right eye 


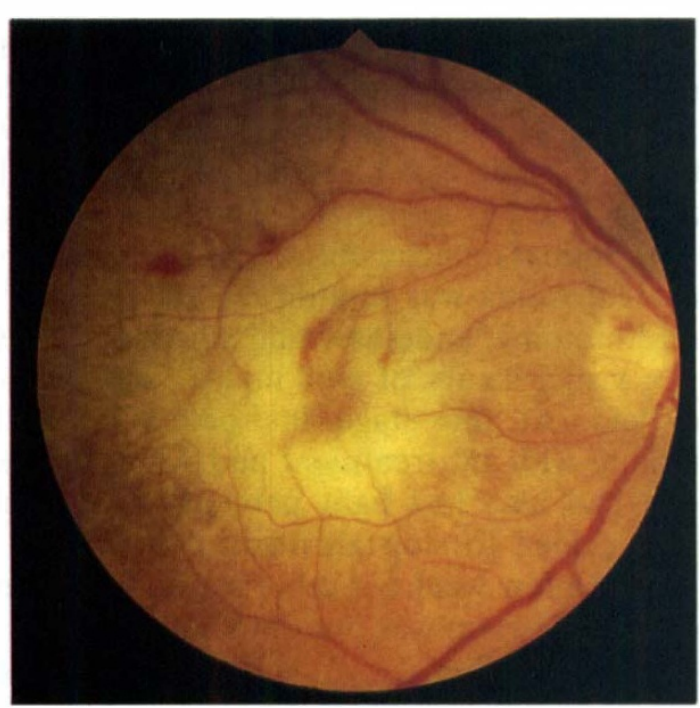

(a)

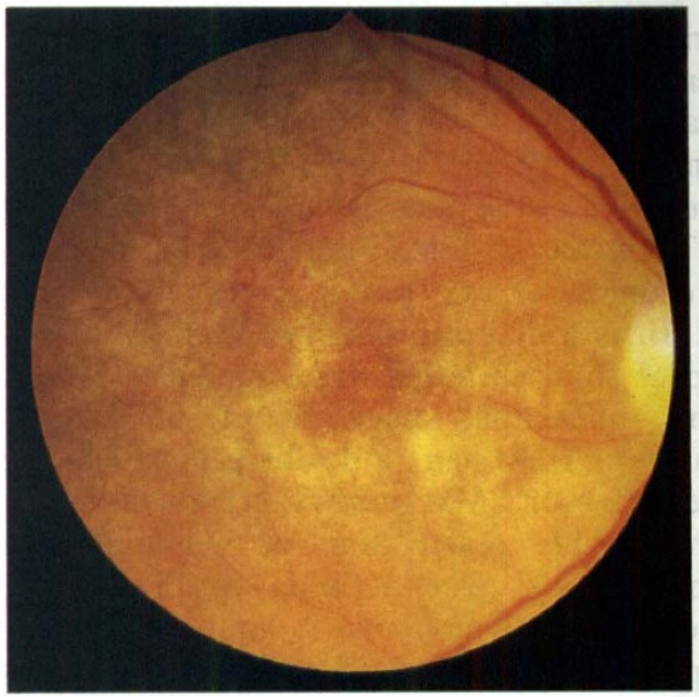

(b)

Fig. 2. Patient 2. (a) Central area of outer retinitis, similar to the one seen in the left eye of the patient 1. (b) Central area of retinitis in the right eye after 13 days of treatment. The lesion is showing signs of improvement with a more granular appearance.

after a few days, at the same time as a space of nonopacified ('normal'-looking) retina could be seen along the retinal vessels. The left CMV retinitis improved and the dose of ganciclovir was reduced to maintenance (one daily dose, 7 days per week) after 3 weeks. There were no signs of outer retinitis in this eye.

The macular retinitis in the right eye showed improvement after 13 days of therapy (Fig. 2b), but the peripheral retinitis progressed with the development of multiple retinal breaks which led to inferior and, subsequently, total retinal detachment. In view of the poor visual prognosis in this eye, retinal reattachment surgery was not undertaken. The left CMV retinitis became quiescent and prophylactic laser photocoagulation was applied around the areas of scar due to the presence of a still attached vitreous and retinal traction in those areas. The patient continued taking IV acyclovir indefinitely $(10 \mathrm{mg} /$ $\mathrm{kg}$ three times per day initially and subsequently twice a day).

The left eye had several recurrences of CMV retinitis which reduced visual acuity to $6 / 36$. His general condition deteriorated progressively and the patient died in December 1993, 1 year after his ophthalmic presentation. Up to his death there were no signs of PORN in the left eye.

\section{METHOD FOR DETECTION OF HERPES VIRAL DNA IN VITREOUS FLUID}

The nested polymerase chain reaction (PCR) method used for the detection of viral DNA sequences from CMV gene gB, VZV gene 29 and HSV-1 gD has been described previously ${ }^{6}$ and was applied to the detection of cell-free vitreous samples from the patients described. The method utilises oligonucleotide primers adapted from those reported by Darlington et al. ${ }^{7}$ Mahalingham et al. ${ }^{8}$ and Aurelius et al. ${ }^{9}$ The first-round reaction mix contained $10 \mathrm{mM}$ Tris- $\mathrm{HCl} \mathrm{pH} 8.3,50 \mathrm{mM} \mathrm{KCl}, 1.5$ $\mathrm{mM} \mathrm{MgCl} 2,1$ unit of Taq DNA polymerase (Perkin Elmer Cetus, ILS Ltd, London), $200 \mu \mathrm{M}$ of each dNTP, $100 \mathrm{ng}$ of each 'outer' primer and $1 \mu \mathrm{l}$ of vitreous supernatant. After an initial 5 minute denaturation at $95^{\circ} \mathrm{C}, 35$ cycles of $95^{\circ} \mathrm{C}$ for 2 minutes $50-60^{\circ} \mathrm{C}$ for 2 minutes and $72{ }^{\circ} \mathrm{C}$ for 1 minute were carried out, followed by a 7 minute extension at $72{ }^{\circ} \mathrm{C}$ using an automated thermal cycler. The reaction conditions for the second round of PCR were as for the first except that the mix contained $100 \mathrm{ng}$ of each 'inner' primer pair and $1 \mu \mathrm{l}$ of the first-round product. Twenty-five cycles were sufficient for the detection of the second-round product. First- and second-round products were analysed by electrophoresis using a $2 \%$ agarose gel, and products were visualised at $302 \mathrm{~nm}$ after staining by ethidium bromide.

\section{DISCUSSION}

The progressive outer retinal necrosis syndrome (PORN), as recently described in two reports, ${ }^{4,5}$ appears to be caused by VZV. It is a new disease characterised by early macular retinitis in the presence of little or no intraocular inflammation. There is rapid progression with the development of lesions in the mid and peripheral retina. The optic nerve may become involved at any stage of the disease. With progression there is clearing of areas along retinal vessels, which has been described as a 'cracked mud' appearance by some authors. ${ }^{5}$ Retinal 
vasculitis appears quite late in the disease course. The appearance is quite distinct from acute retinal necrosis (ARN), in which VZV has also been implicated, and which has also been reported in immunocompromised patients, ${ }^{10,11}$ including those with AIDS. ${ }^{11,12}$ In ARN the striking feature is a peripheral retinitis which becomes confluent and is usually associated with intense vitreous and anterior chamber reaction even if the patient is immunosuppressed.

The reason for a different presentation in the PORN cases compared with the cases of ARN is not clear. It may be that there is a more profound degree of immunosuppression or a different strain of VZV. The two patients described in this report had very low levels of circulating peripheral blood $\mathrm{CD}^{+} \mathrm{T}$ lymphocytes, as in previous reports. ARN is sufficiently infrequent in patients with AIDS to not allow any conclusions to be drawn regarding peripheral $\mathrm{CD}^{+} \mathrm{T}$ lymphocyte numbers in these patients. It is possible that a higher level of immunity may be associated with a more aggressive immune reaction producing the clinical syndrome of ARN, while patients with more profound immunosuppression in AIDS may result in the features of PORN. Some cases of ARN described in immunocompetent hosts follow a less aggressive course, with limited peripheral retinitis and good visual outcome,${ }^{13}$ which could be due to infection with a different strain of virus. ARN associated with varicella has a better prognosis. $^{14}$

The first patient was initially managed with IV foscarnet $(90 \mathrm{mg} / \mathrm{kg}$ twice a day) and IV acyclovir, using the recommended clinical dose $(10 \mathrm{mg} / \mathrm{kg}$ three times per day). The decision to increase the dose of acyclovir to twice the conventional level was made as the normal level of acyclovir therapy had proved ineffective in preventing progression of the disease in other patients (Todd Margolis, personal communication). It is possible that the retinal ischaemia engendered by this infection precludes the achievement of therapeutic levels of acyclovir in the retina. The first patient with bilateral disease at presentation had a good initial response to the higher level of IV acyclovir. This patient deteriorated only when therapy was changed to the oral route of administration. The second patient was treated intravenously from presentation (12 months) and did not show any signs of zoster retinitis in his left eye until his death. The dose of acyclovir was reduced to the usual levels of therapy after 3 months at the higher dose. The experience from these two patients suggests that this higher level of acyclovir might be capable of controlling the progression of the zoster retinitis, but further experience is obviously required. In a series of five cases of presumed varicella-zoster retinopathy recently reported, the authors have also used IV acyclovir, both in normal and in high concentrations $(14 \mathrm{mg} / \mathrm{kg}$ and $22 \mathrm{~g} / \mathrm{kg}$ three times per day). ${ }^{15}$ Three of their patients received IV foscarnet, and in one of them IV ganciclovir was also used. They report stabilisation of the disease in a median time of 51 days, but similar to our experience there was recurrence of the disease once oral acyclovir was used as maintenance therapy. It is also important to investigate the role of intravitreal injections of acyclovir and even foscarnet in the control of the acute presentation of the disease. The efficiency of this regimen in preventing the involvement of the second eye in unilateral presenting cases is questionable and the experience from more cases will be necessary to answer this question.

Even though acyclovir has been found to be more active against $\mathrm{HSV}$ types 1 and $2\left(\mathrm{ID}_{50} 0.1-1.6 \mu \mathrm{M}\right)$ than VZV $\left(\mathrm{ID}_{50} 3-4 \mu \mathrm{M}\right)$ in vitro, ${ }^{16}$ it is recognised as the drug of choice for the treatment of herpes zoster in immunocompromised patients. ${ }^{17,18}$ Recurrence has been reported in patients who switched from intravenous to oral medication, as well as in situations of tapering of oral therapy, ${ }^{15}$ as observed in our first patient. These patients require chronic suppressive antiviral therapy which carries the potential risk for the development of acyclovirresistant varicella-zoster strains, necessitating the use of other antiviral drugs such as foscarnet. ${ }^{19}$ In the search for new antiviral drugs efforts have been concentrated in the development of increased bioavailability prodrugs such as famciclovir. This prodrug of penciclovir is converted to the active drug once absorbed, enabling high serum levels, and potentially increasing efficacy in herpes simplex and VZV-induced diseases. ${ }^{20}$ The benefits of using this drug in cases of PORN have yet to be evaluated.

Rapid control of the progression of the infection is of great importance due to the destructive nature of the inflammation and the high propensity for the retina to detach. Early introduction of high-dose IV acyclovir, especially in unilateral cases, may offer the best treatment option available at present. Such high levels of IV acyclovir demand close monitoring of renal function, and patients need an increase in total fluid intake. Intravitreal injection of antiviral drugs to avoid drug-delivery problems due to ischaemia could also be very important in the management of these cases.

S.D.S. is partially supported by the Ahmanson Foundation, Los Angeles.

Key words: Acute Retinal Necrosis, AIDS, Retinitis, Varicella zoster virus.

\section{REFERENCES}

1. Culbertson WW. Infections of the retina in AIDS. Int Ophthalmol Clin 1989;29:108-18.

2. Holland GN. Acquired immunodeficiency syndrome 
and ophthalmology: the first decade. Am J Ophthalmol 1992;114:85-95.

3. Reese L, Sheu MM, Lee F, Kaplan HJ, Nahmias A. Intraocular antibody production suggests herpes zoster is only one cause of acute retinal necrosis (ARN). Invest Ophthalmol Vis Sci 1986;27(Suppl):12.

4. Forster DJ, Dugel PU, Frangieh GT, Liggett PE, Rao NA. Rapidly progressive outer retinal necrosis in the acquired immunodeficiency syndrome. Am J Ophthalmol 1990;100:341-8.

5. Margolis TP, Lowder CY, Holland GN, Spaide RF, Logan AG, Weissman SS, et al. Varicella-zoster virus retinitis in patients with the acquired immunodeficiency syndrome. Am J Ophthalmol 1991;112:119-31.

6. Mitchell SM, Fox JD, Tedder RS, Gazzard BG, Lightman SL. Vitreous sampling and viral genome detection for the diagnosis of viral retinitis in patients with AIDS. J Med Virol 1994;43:336-40.

7. Darlington J, Super M, Patel K, Grundy JE, Griffiths PD, Emery VC. Use of the polymerase chain reaction to analyse sequence variation in major neutralising epetope of glycoprotein B (gp58) in clinical isolates of human cytomegalovirus.' J Gen Virol 1991;72:1985-9.

8. Mahalingam R, Wellish M, Wolf W, Dueland AN, Cohrs R, Vafai A, Gilden D. Latent varicella-zoster viral DNA in the human trigeminal and thoracic ganglia. N Engl J Med 1990;323:627-31.

9. Aurelius E, Johansson B, Skoldenberg B, Staland A, Forsgren M. Rapid diagnosis of herpes simplex encephalitis by nested polymerase chain reaction of cerebrospinal fluid. Lancet 1991;337:189-92.

10. Friberg TR, Jost BF. Acute retinal necrosis in an immunosuppressed patient [letter]. Am J Ophthalmol 1984;98:515-7.
11. Jabs DA, Schachat AP, Liss R, Knox DL, Michels RG. Presumed varicella zoster retinitis in immunocompromised patients. Retina 1987;7:9-13.

12. Chess J, Marcus DM. Zoster-related bilateral acute retinal necrosis syndrome as presenting sign in AIDS. Ann Ophthalmol 1988;20:421-8.

13. Matsuo T, Nakayama T, Koyama T, Koyama M, Matsuo N. A proposed mild type of acute retinal necrosis syndrome. Am J Ophthalmol 1988;105:579-83.

14. Culbertson WW, Brod RD, Flynn HW Jr, Taylor BC, Brod BA, Lightman DA, Gordon G. Chickenpoxassociated acute retinal necrosis syndrome. Ophthalmology 1991;98:1641-6.

15. Johnston WH, Holland GN, Engstrom RE Jr, Rimmer S. Recurrence of presumed Varicella-zoster retinopathy in patients with acquired immunodeficiency syndrome. Am J Ophthalmol 1993;116:42-50.

16. Reese ER, Sentochnik DE, Douglas RG Jr, Betts RF/ Antiviral agents. In: Handbook of Antibiotics. Bostons Little, Brown, 1988:241.

17. Gnann JW Jr. New antivirals with activity against varicella-zoster virus. Ann Neurol 1994;35 (Suppl) S69-72.

18. Balfour HH Jr. Varicella-zoster virus infections in the immunocompromised host: natural history and treat ment. Scand J Infect Dis Suppl 1991;80:69-74.

19. Safrin S, Berger TG, Gilson I, Wolfe PR, Wofsy CF Mills J, Biron KK. Foscarnet therapy in five patien with AIDS and acyclovir-resistant varicella-zoster vir infection. Ann Intern Med 1991;115:19-21.

20. Sasadeusz JJ, Sacks SL. Systemic antivirals in herpe virus infections. Dermatol Clin 1993;11:171-85. 